\title{
On the Determination and Constancy of the Solar Oblateness
}

\author{
M. Meftah ${ }^{1}$ A. Irbah ${ }^{1}$ - A. Hauchecorne ${ }^{1}$ - T. Corbard ${ }^{2}$. \\ S. Turck-Chièze ${ }^{3}$ J.-F. Hochedez ${ }^{1,4} \cdot$ P. Boumier ${ }^{5}$.

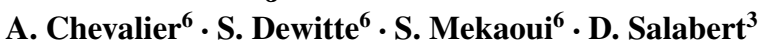

Received: 19 November 2013 / Accepted: 21 January 2015 / Published online: 11 February 2015

(C) The Author(s) 2015. This article is published with open access at Springerlink.com

\begin{abstract}
The equator-to-pole radius difference ( $\left.\Delta r=R_{\mathrm{eq}}-R_{\mathrm{pol}}\right)$ is a fundamental property of our star, and understanding it will enrich future solar and stellar dynamical models. The solar oblateness $\left(\Delta_{\odot}\right)$ corresponds to the excess ratio of the equatorial solar radius $\left(R_{\mathrm{eq}}\right)$ to the polar radius $\left(R_{\mathrm{pol}}\right)$, which is of great interest for those working in relativity and different areas of solar physics. $\Delta r$ is known to be a rather small quantity, where a positive value of about 8 milli-arcseconds (mas) is suggested by previous measurements and predictions. The Picard space mission aimed to measure $\Delta r$ with a precision better than 0.5 mas. The Solar Diameter Imager and Surface Mapper (SODISM) onboard Picard was a Ritchey-Chrétien telescope that took images of the Sun at several wavelengths. The SODISM measurements of the solar shape were obtained during special roll maneuvers of the spacecraft by $30^{\circ}$ steps. They have produced precise determinations of the solar oblateness at $782.2 \mathrm{~nm}$. After correcting measurements for optical distortion and for instrument temperature trend, we found a solar equator-to-pole radius difference at $782.2 \mathrm{~nm}$ of $7.9 \pm 0.3$ mas $(5.7 \pm 0.2 \mathrm{~km})$ at one $\sigma$. This measurement has been repeated several times during the first year of the space-borne observations, and we have not observed any correlation between oblateness and total solar irradiance variations.
\end{abstract}

Keywords Solar diameter $\cdot$ Solar oblateness $\cdot$ Solar irradiance $\cdot$ Active regions

M. Meftah

Mustapha.Meftah@latmos.ipsl.fr

1 Université Versailles St-Quentin, Sorbonne Universités, CNRS/INSU, LATMOS-IPSL, UPMC

Université Paris 06, 11 Boulevard d'Alembert, 78280 Guyancourt, France

2 OCA-Observatoire de la Côte d'Azur, Laboratoire Lagrange, CNRS, Université de Nice-Sophia Antipolis, Parc Valrose, 06108 Nice Cedex 2, France

3 SAp/IRFU/CEA, Centre de Saclay, 91191 Gif-sur-Yvette Cedex, France

4 ORB, 3 Avenue Circulaire, 1180 Uccle, Belgium

5 IAS-Institut d'Astrophysique Spatiale, CNRS, Université Paris XI, 91471 Orsay, France

6 IRMB, 3 Avenue Circulaire, 1180 Uccle, Belgium 


\section{Introduction}

The rotation of the Sun leads to a flattening of the polar regions. It is believed that the solar oblateness $\left(\Delta_{\odot}=\left(R_{\mathrm{eq}}-R_{\mathrm{pol}}\right) / R_{\mathrm{pol}}\right)$ results from the internal rotation of the Sun and from its mass distribution. The physical interest in the asphericity is described in e.g. Roxburgh (2001) or Fivian et al. (2008). If the solar surface deformation provides an indirect information on the inner rotation profile and on the distribution of matter, it could also provide information on the internal magnetic field (Duez, Mathis, and Turck-Chièze, 2010) or on the Earth-Sun relationship, which means that the magnitude of the solar oblateness remains an important observable for constraining models of the solar interior and predictions of its variability. It also places constraints on general relativity.

The interest in this knowledge began in the nineteenth century, when Urbain Le Verrier showed that there is a slow precession of Mercury's orbit, i.e. its perihelion rotates around the Sun at about 574 arcseconds per century (Le Verrier, 1859). According to Newton's theory, this value should be 531 arcseconds, i.e. there is a difference of 43 arcseconds between observation and theory. Mercury has a highly elliptical orbit. If the Sun and Mercury were the only objects in the Universe, Mercury's perihelion would remain at the same place at each revolution, but because of the gravitational disturbance between the planets, this is not the case. At each revolution, Mercury's orbit drifts by a few arcseconds. To explain the different results of the calculations of Newton's theory and the observations made by Urbain Le Verrier, astronomers of that era even tried imagining the existence of a planet (Vulcan) between Mercury and the Sun. Finally, Albert Einstein calculated the correction that the general relativity (Einstein, 1916) brings to Newton's theory and justified the 43 arcseconds difference. But the calculation did not take into account the shape of the Sun.

The main reasons are that the oblateness is difficult to measure because its value is very low and because it can be perturbed by the surface magnetic activity, i.e. by sunspots and faculae (Fivian et al., 2008). Based on measurements collected from various instruments over the past 140 years (Rozelot and Damiani, 2011; Damiani et al., 2011), the measured solar equator-to-pole radius difference $\left(\Delta r=R_{\mathrm{eq}}-R_{\mathrm{pol}}\right)$ has generally become smaller over time from 500 mas in 1870 (Poor, 1905) to 7.2 mas in 2012 (Kuhn et al., 2012), mainly due to the instrument precision and data processing. Solar oblateness became of interest some decades ago when it was invoked to challenge the standard Einsteinian general relativity (Brans and Dicke, 1961; Dicke and Goldenberg, 1967). As of today, there are few measurements from space, which are very important, however, because they seem to achieve the required sensitivity in measuring the solar oblateness. Investigating solar oblateness issues (in value and trend) remains a worthwhile task (Rozelot and Fazel, 2013).

The Picard spacecraft was launched in June 2010 and was dedicated to studying the Sun. The Solar Diameter Imager and Surface Mapper (SODISM), a Ritchey-Chrétien telescope onboard Picard (Meftah et al., 2014a), provided full-disk images of the Sun in five narrowwavelength passbands (centered at 215.0, 393.37, 535.7, 607.1, and 782.2 nm). Measurement of the solar asphericity at several wavelengths was achievable with this instrument. However, its precise value required specific modes in the operations to reach an accuracy that allows us to check the different terms of the solar asphericity. The spacecraft maneuver was essential to determining the solar shape in order to remove the effect of instrument optical aberrations. During Picard's measurement campaigns, the whole spacecraft revolved around the Picard-Sun axis. The first roll maneuver completed on September 2010 was made at 12 positions spaced at $30^{\circ}$ roll steps, and we have taken 10 images per wavelength and per step. Such campaigns occurred several times, as shown in this article. Then the procedure was improved, and in May 2011, we achieved each roll step of Picard within an orbit 


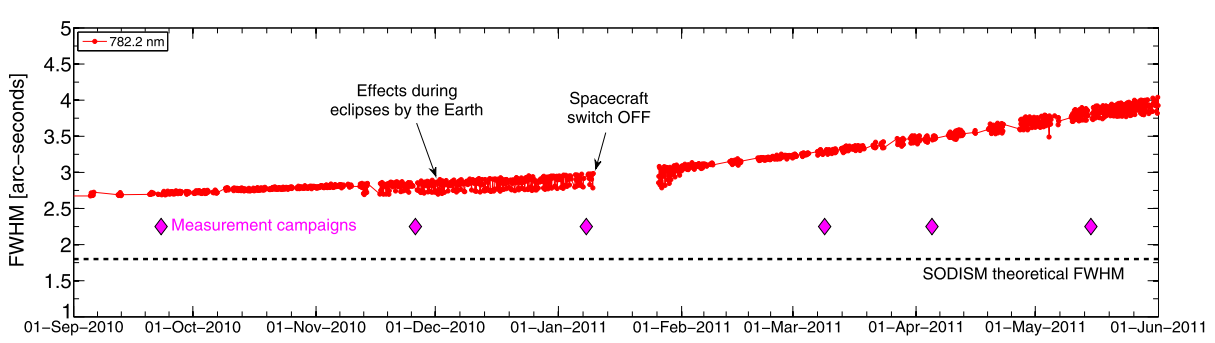

Figure 1 Temporal evolution of the solar limb FWHM at $782.2 \mathrm{~nm}$ measured by SODISM (red circles) during the solar oblateness measurement campaigns. Measurement campaigns at $782.2 \mathrm{~nm}$ are indicated by pink diamonds.

with $\sim 58$ images at $782.2 \mathrm{~nm}$ for the same angular position of the spacecraft. Space is a harsh environment for optics, with many physical interactions leading to potentially severe degradation of the optical performance over time. The type of degradation that can affect SODISM is the degradation of image quality due to a combination of solar irradiation and instrumental contamination. The raw images have revealed some optical aberrations that blur the images (Meftah et al., 2014a) increasingly with time (see Figure 1).

The SODISM point spread function (PSF) and its effects on the solar limb have been studied for different optical configurations, wherein the instrument is diffraction-limited. Thermal gradients in the front window result in a significant evolution of the full width at half maximum (FWHM) of the solar limb's first derivative. At $782.2 \mathrm{~nm}$, the effect is weaker, therefore we focused on this wavelength. However, during each measurement campaign, the degradation of the instrument can be treated as constant. The spacecraft has to complete a roll within an orbit, so there was not enough time to take images at the other wavelengths. Indeed, an image is taken every two minutes to respect the telemetry budget. In addition, the solar radius measurement systematically varies in time and displays a modulation in phase with the orbit, which depends on the wavelength (see Figure 4). The solar radius measured by SODISM is correlated with the front window temperature. This led us to focus on a modification of the solar oblateness procedure (increase the number of measurements and using a single wavelength).

In this article, we report on the solar oblateness measurements of SODISM at $782.2 \mathrm{~nm}$. These measurements were carried out between 2010 and 2011. One of the questions raised is how the solar oblateness changes with respect to the total solar irradiance.

\section{Theoretical Solar Oblateness}

The theoretical solar oblateness, $\Delta_{\odot}$, has been found to be between $6.5 \times 10^{-6}$ and $10.2 \times$ $10^{-6}$ (Rozelot, Godier, and Lefebvre, 2001). If only a rigid rotation in the radiative zone and the differential rotation in the convective zone (Mecheri et al., 2004) are considered, the theoretical value of the solar oblateness is near $9.1 \times 10^{-6}$. In another study, this parameter was found to be between $5.6 \times 10^{-6}$ and $8.4 \times 10^{-6}$ (Fazel et al., 2008). If the rotation of the core is also considered, the value exceeded $8.4 \times 10^{-6}$ (Paterno, Sofia, and di Mauro, 1996). But some deformation coming from the internal magnetic field cannot be excluded, which, depending on its topology, can have a positive or negative supplementary term. Moreover, Gough's (2012) discussion of recent measurements shows that the Sun appears to be closer to spherical than current understanding predicts. 


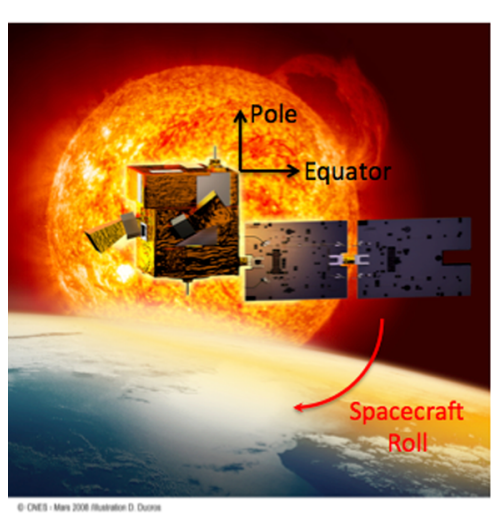

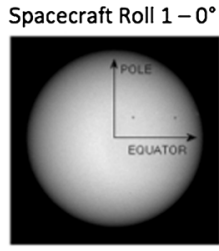

Spacecraft Roll $7-180^{\circ}$

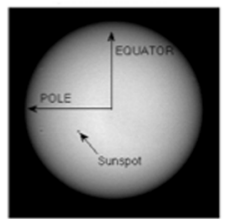

Spacecraft Roll $4-90^{\circ}$

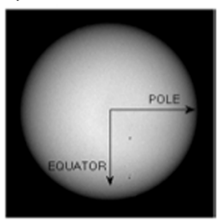

Spacecraft Roll $10-270^{\circ}$

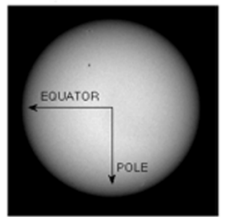

Figure 2 During solar oblateness measurement campaigns, the Picard spacecraft revolves around the $P i$ card-Sun axis by steps of $30^{\circ}$ from north to west (clockwise). Solar images are taken for each roll step of the spacecraft.

It is clear that the solar oblateness is low, therefore it is difficult to measure precisely. An accurate measurement will bring new information on some processes that are difficult to obtain directly, such as the potential presence of magnetic field in the radiative zone, some effects on the orbital motions of the planets, or their influence on the solar shape. Moreover, the wavelength dependence of the solar oblateness is not yet obtained, only sparse information with totally different approaches can be found in the literature.

\section{Method for Determining the Solar Oblateness}

We describe in this section the method used to estimate the solar equator-to-pole radius difference in the solar continuum. After analyzing the data acquired by the telescope, we identified six sequences of images at $782.2 \mathrm{~nm}$ taken during specific campaigns with a sufficient level of quality to determine the solar oblateness in value and trend. The optical distortion of SODISM solar images is a few orders of magnitude larger than what we would like to extract. Therefore the solar oblateness is extracted from different sequences of images taken at $782.2 \mathrm{~nm}$ during specific campaigns, whose times are indicated in Figure 1. In addition, we have increased the number of images during the May 2011 campaign to improve the statistics of the measurement and to reduce the error bar.

The solar oblateness measurement campaign allows determining and removing the effect of distortion in images and temperature effects of the instrument. In this mode, the spacecraft rotates around its axis directed at the Sun, taking 12 positions separated by $30^{\circ}$ (Figure 2 ), thus the polar and equatorial diameters of the Sun successively take the 12 orientations on the charge-coupled device (CCD) image. The CCD detector array has $2048 \times 2048$ pixels of $13.5 \mu \mathrm{m}$ pitch. A solar oblateness measurement campaign took less than two days, therefore the drift of the instrument parameters may be neglected. As shown in Figure 1, the evolution of the solar limb at FWHM is slow and persistent, which justifies our hypothesis. This section describes the method used to obtain the necessary parameters (solar radius, distortion, and temperature correction) to determine the solar equator-to-pole radius difference in seven steps. 

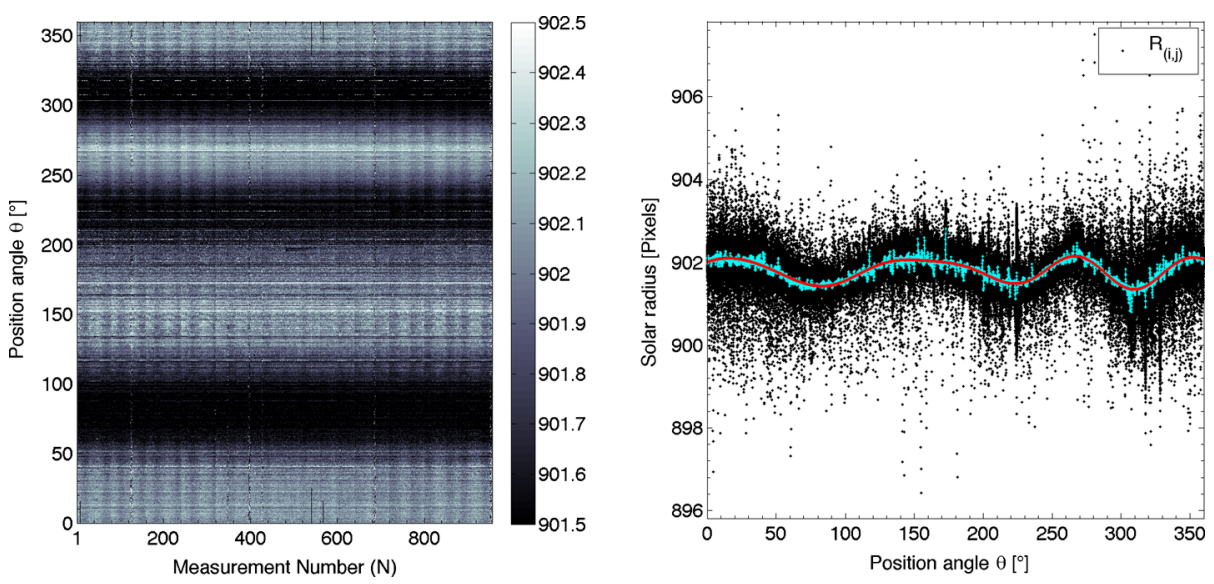

Figure 3 (Left) Measured solar radii $R_{(i, j)}$ where the units of the color bar are in pixels. In this sample, the roughly 900 images are distributed over 12 sections containing the images for each orientation angle. (Right) Solar radii (black dots) vs. CCD angle in a polar coordinate system. Average limb position (cyan dots) $v s$. CCD angle plotted in pixel units. Distortion curve obtained by fitting a sum of sines (the correlation coefficient, $R^{2}$, is 0.975 ).

Solar Radius Determination We define the solar radius of each image by the inflection point position (IPP) of the solar-limb profiles taken at different angular positions of the image $\left(N_{\theta}\right)$. Solar limb profiles are obtained through creating a line associated with virtual pixels that is oriented in the desired angular position. Each virtual pixel of the line is located in an area of four physical pixels on the CCD. The intensity of the virtual pixel is calculated using a bilinear interpolation of the four pixels. Then, the resolution of the line associated with the virtual pixels is oversampled (using the Fourier transform). Thus, we obtain a solar limb profile observed by the SODISM instrument at a given angular position. From this solar limb profile, we can determine the best polynomial fit (of fifth order for this application) and obtain its point of inflection. After computing 4000 IPP according to the different angular positions, we can obtain the best fit of the solar contour (ellipse) using the least-squares method. From this method, we obtain a result with a resolution of sub-pixel level. Thus, we can obtain the location of the center of the solar image. We repeat all the IPP calculation until the center of the solar image is found at a precision better than 0.05 pixel (criterion used in this analysis) after a few successive iterations. In summary, the IPP is obtained by the passage through zero of the solar limb second derivative. The contour of all the IPP is calculated independently for each of the $N$ images. Then, we describe the series of solar radii measured $\left(R_{(i, j)}\right)$ by a $N \times N_{\theta}$ matrix (see Figure 3 , left panel), where all the radii are calculated in the $N_{\theta}$ directions.

Mean Solar Radius Evolution A mean solar radius is obtained by averaging the calculated radii over all the azimuthal angles within one image. Thus, we can obtain the evolution of the mean measured solar radius over time (Figure 4). The bad radius measurements are due to solar images recorded when the spacecraft crosses the South Atlantic Anomaly (SAA). The SAA is the region where the Earth's inner Van Allen radiation belt makes its closest approach to the planet's surface. The result is that for a given altitude, the radiation intensity is higher over this region than elsewhere. The SODISM CCD camera is strongly affected by the particles when the spacecraft crosses the SAA. The bad images, acquired when the spacecraft crosses the SAA, are ignored. 


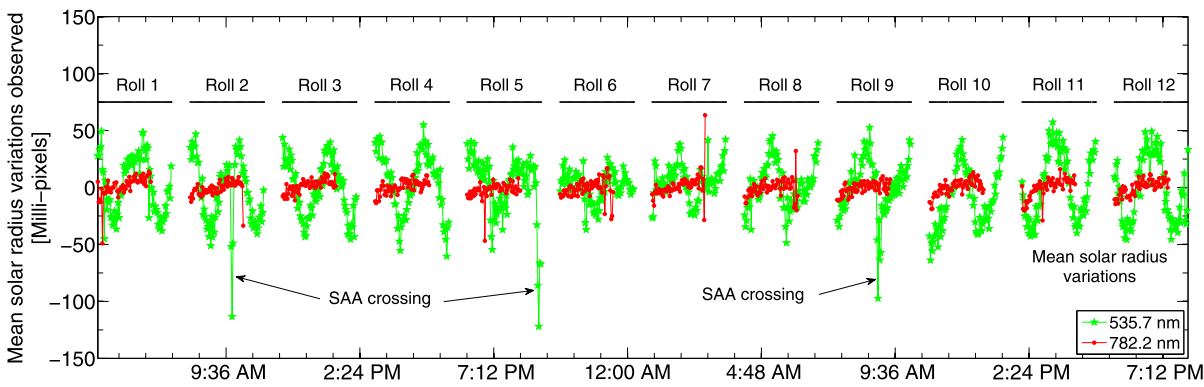

Figure 4 Evolution of the mean solar radius (green curve with stars for $535.7 \mathrm{~nm}$ and red curve with circles for $782.2 \mathrm{~nm}$ measurements) during a solar oblateness campaign and effect of the SAA on SODISM images. The images taken for each roll of the spacecraft are represented by the 12 black segments. These curves display a modulation in phase with the rotation during the roll, which depends on the wavelength. At $782.2 \mathrm{~nm}$, the effect is weaker, which minimizes the correction associated to the factor $k_{(j)}$ and focuses our interest on this wavelength.

Image Distortion Definition The inferred average solar radius as a function of offset angle with respect to the CCD indicates that the images of SODISM are significantly distorted (Figure 3, right panel). The residual pattern (after subtracting the largely dominant sphericity) reveals a triangular trefoil shape. The distortion curve is obtained from the mean calculated solar radius along the same angle. After completing this operation, we fit a sum of sines through the cloud of points. Finally, we verify the goodness of the fit ( $R^{2}$ correlation coefficient). Thereafter, we assume that the underlying solar profile is the same for all polar angles on the solar disk (in contrast to Kuhn et al. 1998). Indeed, we calculate semidiameters to better characterize the center of the solar image and to have more accurate results.

Data Correction After completing the first three steps, we correct our data using Equations (1) and (2) below optical distortion and thermal effects, which are reversible (i.e. cause no degradation of the telescope) over short periods.

$$
\langle R\rangle=\frac{1}{N \times N_{\theta}} \sum_{m=1}^{N} \sum_{n=1}^{N_{\theta}} R_{(m, n)}
$$

and

$$
\begin{aligned}
R c_{(i, j)}= & R_{(i, j)}+\left(\langle R\rangle-\frac{1}{N_{\theta}} \sum_{n=1}^{N_{\theta}} R_{(i, n)}\right) \\
& +G \times\left(T_{(i)}-\frac{1}{N} \sum_{m=1}^{N} T_{(m)}\right)+k_{(j)} \times\left(\langle R\rangle-\frac{1}{N} \sum_{m=1}^{N} R_{(m, j)}\right),
\end{aligned}
$$

where $R c_{(i, j)}$ is the corrected solar radius at one astronomical unit ( $\left.1 \mathrm{AU}\right), R_{(i, j)}$ is the calculated solar radius corrected at 1 AU after IPP determination, $N$ is the number of images, and $N_{\theta}$ is the number of angles of the image. $G$ is the temperature coefficient of the CCD, and $T_{(i)}$ is the CCD temperature. The temperature of the front window $\left(T_{f(i)}\right)$ during the time where an image is obtained is linearly regressed to all the inferred radii in all directions with respect to the CCD orientation, and $k_{(j)}$ is the slope of the linear regression.

The CCD temperature changes during the measurements. It is necessary to correct for this to take into account the evolution of the instrument plate scale (Meftah et al., 2014c). 

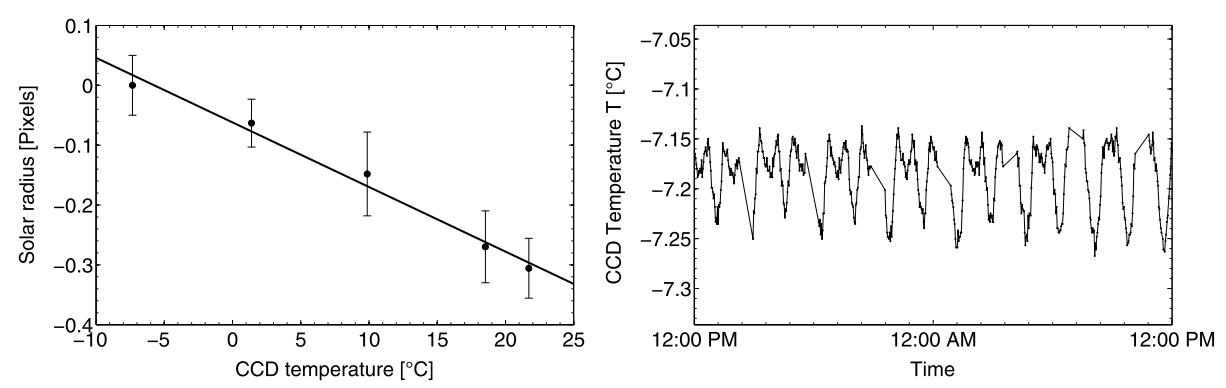

Figure 5 (Left) Relation between the CCD temperature and the solar radius obtained during a specific calibration test. The temperature coefficient of the $\operatorname{CCD}(G)$, determined from the slope, is equal to -0.01079 pixel $^{\circ} \mathrm{C}^{-1}$. (Right) $\mathrm{CCD}$ temperature evolution during a set of measurements (nominal temperature regulation).

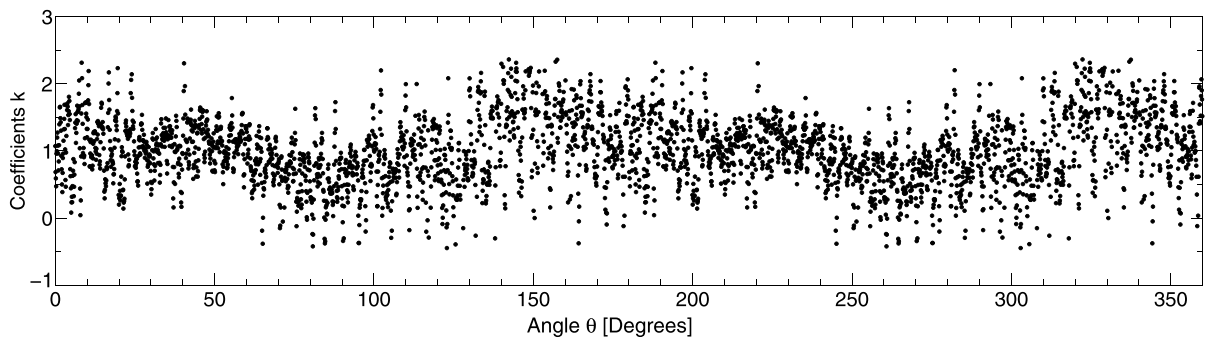

Figure 6 Coefficients, $k_{(j)}$, of the linear regression as a function of the image angle.

We calibrated the instrument plate scale (through the correction parameter $G$, see Figure 5, left panel) as a function of the CCD temperature evolution (Figure 5, right panel). This correction allows taking into account the thermal expansion of the CCD pixel size. However, higher order terms can be neglected, such as cross terms depending on the factor $G$ (the CCD temperature does not vary by much, as seen in the right panel of Figure 5).

Another correction is associated with the relation between the SODISM front window temperature (or mean solar radius) and the solar radii associated with an image. Indeed, during the orbit, the SODISM mean solar radius measurement and the front window temperature show the same behavior (Meftah et al., 2014a) as a result of the terrestrial atmospheric radiation, which affects the observations (Irbah et al., 2012; Meftah et al., 2013). For this reason, we determine the slope of the linear regression $k_{(j)}$ between the front window temperature and the solar radii (Figure 6).

Change of Reference from the CCD to the Solar Frame The rotation of the spacecraft is from north to west (clockwise). Equation (3) below describes the transformation of the corrected solar radii from the CCD frame (Figure 7, left panel) to the solar fixed frame (right panel) during the 12 positions taken by the spacecraft (Equation (4))

$$
\begin{aligned}
& \theta(\operatorname{Sun})_{(i, j)}=\theta_{(j)}+\left(\operatorname{Roll}_{(i)}-1\right) \times 30, \\
& 1 \leqslant \operatorname{Roll}_{(i)} \leqslant 12 .
\end{aligned}
$$

Apparent Solar Oblateness Determination To determine the apparent solar oblateness, we fit the corrected solar radii (solar fixed frame) with a sum of sine and cosine functions 

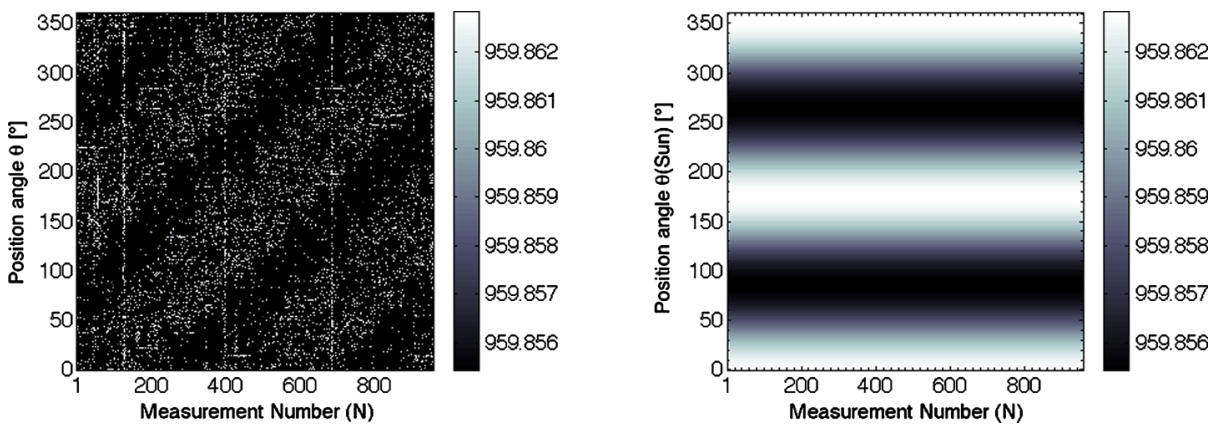

Figure 7 (Left) Corrected solar radii $R c_{(i, j)}$. (Right) Apparent solar equator-to-pole radius difference $\left(\Delta r_{\mathrm{a}}\right)$ obtained by fitting a sum of sine and cosine functions. The right panel highlights the change of reference from the CCD to the solar frame.
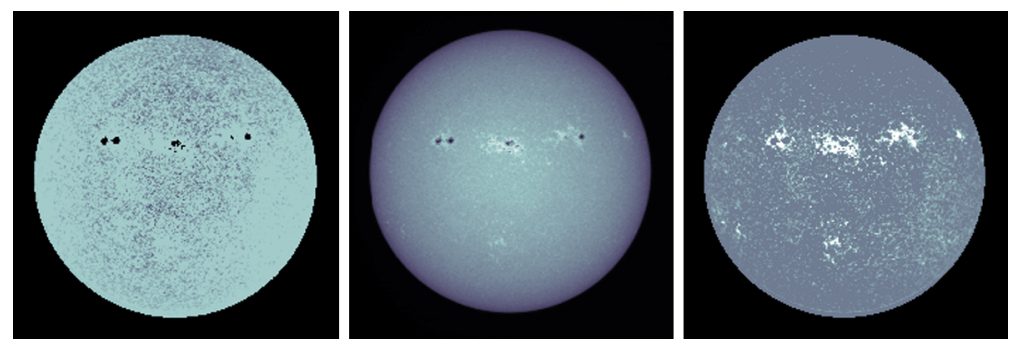

Figure 8 (Central panel) SODISM images at $393.37 \mathrm{~nm}$ (Ca II K) showing the counteracting effects on solar irradiance of sunspots and pores and bright network and faculae. (Left panel) Result of applying the method for extracting sunspots and pores from SODISM images (black). (Right panel) Result of applying the method for extracting network and faculae (white).

(Figure 7, right panel). As a first step, we inspected images at $393.4 \mathrm{~nm}$ (Ca II K) provided by the Precision Solar Photometric Telescope (PSPT) (http://lasp.colorado.edu/pspt_access/), as SODISM images at $393.37 \mathrm{~nm}$ were analyzed before and after the solar oblateness measurement campaign (no SODISM Ca II K full-disk image was taken during the campaign). We have developed a method to isolate and extract all bright and dark active region features from each image, which provide positive and negative intensity contributions to the spectral solar irradiance, respectively. For every image, a model of the quiet Sun is built. A mean limb-darkening function of the Sun is computed using several cuts of the image. The quiet Sun is then built with this mean limb, and we subtract it from the original image. All positive and negative values correspond to the bright and dark features of active regions.

Figure 8 shows the result obtained when we apply our processing method to the recorded images. Thus, there is a method to deal with the cross-talk of intensity variations (e.g. active regions, faculae, etc.) with the inferred limb location (by masking the data using the $\mathrm{Ca}$ II $\mathrm{K}$ images). The final fit of the apparent solar oblateness does not take into account the affected solar limb portions. Then, we verify the goodness of fit $\left(R^{2}\right.$ correlation coefficient) and obtain the uncertainty of the measurements (95\% confidence bounds, or $2 \sigma$ ). The confidence bounds for fitted coefficients are given by $C=b \pm t \times S^{0.5}$, where $b$ are the coefficients produced by the fit, $t$ depends on the confidence level and is computed using the inverse of Student's $t$ cumulative distribution function, and $S$ is a vector formed with the diagonal elements from the estimated covariance matrix of the coefficient estimates. To 

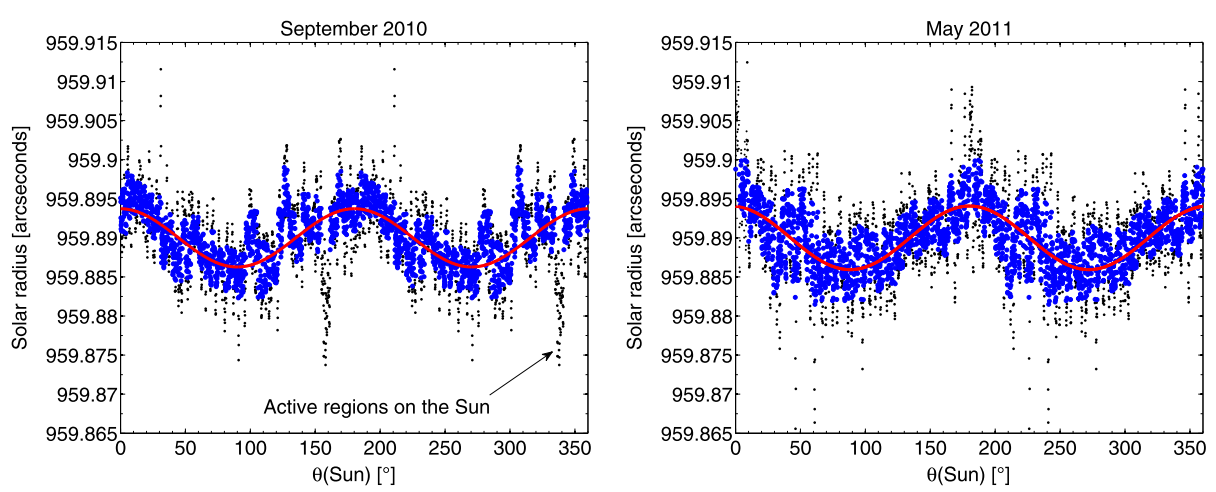

Figure 9 At $782.2 \mathrm{~nm}$, the mean solar radius at $1 \mathrm{AU}$ is about 959.89 arcseconds $(\langle r\rangle)$. (Left) Active regions on the Sun are places where the solar magnetic field is especially strong. The IPP moves about 20 mas, which generates the smaller solar radius observed. The data obtained without correction are plotted with black dots. The filtered data are represented with blue circles. In September 2010, the shape of the Sun is given by the continuous red line (sinusoidal fit). (Right) Shape of the Sun in May 2011. $0^{\circ}$ and $180^{\circ}$ refer to the solar equatorial radius, whereas $90^{\circ}$ and $270^{\circ}$ refer to the solar polar radius.

complete this step, we calculate the root-mean-square-error (Rmse) of the solar oblateness fit (Equation (5)). The use of Rmse is an excellent general-purpose error metric for numerical predictions

$$
\text { Rmse }=\sqrt{\frac{1}{n} \sum_{k=1}^{n}\left(Y d a t a_{(k)}-Y f i t_{(k)}\right)^{2}} .
$$

Heliographic Latitude $\left(\boldsymbol{B}_{\mathbf{0}}\right)$ We correct for the tilt of the ecliptic with respect to the solar equatorial plane (Equation (6)). For an ellipsoid of revolution, the apparent oblateness will decrease when the line of sight is not perpendicular to the rotation axis. To first order, the apparent equator-to-pole radius difference $\left(\Delta r_{\mathrm{a}}\right)$ seen from an heliographic latitude $B_{0}$ is related to the true solar equator-to-pole radius difference $(\Delta r)$. During the year, the heliographic latitude of Picard stays within $\pm 7.25^{\circ}$, which leads to a maximum reduction for $\Delta r_{\mathrm{a}}$ of about $1.6 \%$ around the two equinoxes. Thus, for a true $\Delta r$ around 8 mas and $B_{0}$ within $\pm 7.25^{\circ}$, this leads to a maximum reduction of about 0.13 mas for $\Delta r_{\mathrm{a}}$ around the two equinoxes

$$
\Delta r=\frac{\Delta r_{\mathrm{a}}}{\cos ^{2} B_{0}}
$$

\section{Results and Discussion}

After this processing (see Section 3), we determine the solar oblateness at $782.2 \mathrm{~nm}$. After correcting the measurements for optical distortion and for instrument temperature trend, we find an apparent solar equator-to-pole radius difference $\left(\Delta r_{\mathrm{a}}\right)$ of $7.45 \pm 0.35$ mas $(2 \sigma)$ for September 2010 (left panel in Figure 9), and of $7.82 \pm 0.29$ mas $(2 \sigma)$ for May 2011 (right panel in Figure 9) using a sinusoidal fit to the data. The apparent solar shape $(r(\theta))$ can be also expressed using Legendre polynomials $\left(P_{l}\right)$, as shown in Equation $(7)$ below. The 
solar shape is often treated with this method. Thus, we can determine $\Delta r_{\mathrm{a}}$ from $C_{2}$ and $C_{4}$ coefficients (Equation (8)).

$$
\begin{aligned}
r(\theta)= & \langle r\rangle \times\left(1+\sum_{l=2,4} C_{l} \times \bar{P}_{l}(\cos (\Theta))\right) \\
= & \langle r\rangle \times\left(1+C_{2} \times \frac{1}{4} \times(3 \times \cos (2 \Theta))\right) \\
& +\langle r\rangle \times\left(C_{4} \times \frac{1}{64} \times(35 \times \cos (4 \Theta)+20 \times \cos (2 \Theta))\right)
\end{aligned}
$$

and

$$
\Delta r_{\mathrm{a}}=r\left(\frac{\pi}{2}+\varphi\right)-r(\varphi)=\langle r\rangle \times\left(-\frac{3}{2} C_{2}-\frac{5}{8} C_{4}\right),
$$

where $\Theta=\theta-\varphi, \theta$ is the heliographic colatitude, $\varphi$ is a phase allowing us to take into account a pointing uncertainty during the roll, $\langle r\rangle$ is the mean solar radius at $1 \mathrm{AU}, \bar{P}_{l}$ is the Legendre polynomial of degree $l$ shifted to have zero mean $\left(\bar{P}_{l}=P_{l}-\left\langle P_{l}\right\rangle\right), C_{2}$ and $C_{4}$ are the quadrupole and hexadecapole coefficients.

From this method associated with the polynomial expansion of the solar radius contour, we find from the sequence of measurements of September 2010 the quadrupole term as $C_{2}=(-5.24 \pm 0.23) \times 10^{-6}$ and the hexadecapole term as $C_{4}=(+0.26 \pm 0.25) \times 10^{-6}$ $(2 \sigma)$, which yield $\Delta r_{\mathrm{a}}=7.39$ mas. Similarly, we obtain $C_{2}=(-5.98 \pm 0.33) \times 10^{-6}$ and $C_{4}=(+1.38 \pm 0.40) \times 10^{-6}(2 \sigma)$ in May 2011, which yield $\Delta r_{\mathrm{a}}=7.78$ mas. A more complete analysis in Legendre polynomials, with a better instrumental knowledge, is required to search for some manifestation of the internal magnetic field or the latitudinal rotation. We have preferred in this initial study to only concentrate on the solar equator-to-pole radius difference. We note that the results are not significantly affected by these different fits (sinusoidal or Legendre polynomial fits), as shown below.

To perform these analyses, we used Level 1 data products (corrected raw images for dark current and flatfield). Indeed, the image data of the telescope require corrections for their imperfections of instrumental origin (dark current, flatfield, etc.). The image data of SODISM require dark-signal correction and hot-pixel identification (Hochedez et al., 2014). The images at $782.2 \mathrm{~nm}$ are also strongly dominated by an interference pattern in the surface layer of the CCD that disappears after flatfield correction (Figure 10). All raw images (Level 0 data products) have been corrected for dark current using the method proposed by Hochedez et al. (2014) and flatfield using the method proposed by Kuhn, Lin, and Loranz (1991). If we forego these corrections to our data (from Level 0 to Level 1), we introduce a systematic bias in our results $(\sim-0.8$ mas). The uncertainty in the knowledge of the plate scale (Meftah et al., 2014c) slightly affects our solar oblateness results (less than 0.01 mas). We also note that the precision of the telescope pointing is better than \pm 0.2 arcsecond root-mean-square error during the whole solar oblateness measurement campaign. Thus, the effect on the solar oblateness measurement associated with the instrument pointing is negligible.

The two sets of measurements (September 2010 and May 2011) differ significantly in number of images $(N)$ and performance of the instrument for a given period (images are increasingly blurred, see Figure 1). Table 1 summarizes the main characteristics and results of these two sequences. The goodness of fit ( $R^{2}$ correlation coefficient) is better for the set of measurements made in May 2011, despite the degradation of the instrument (compensated for by the number of images taken). This is because the degradation does not affect the reproducibility. The May 2011 sequence takes better advantage of the latter. 

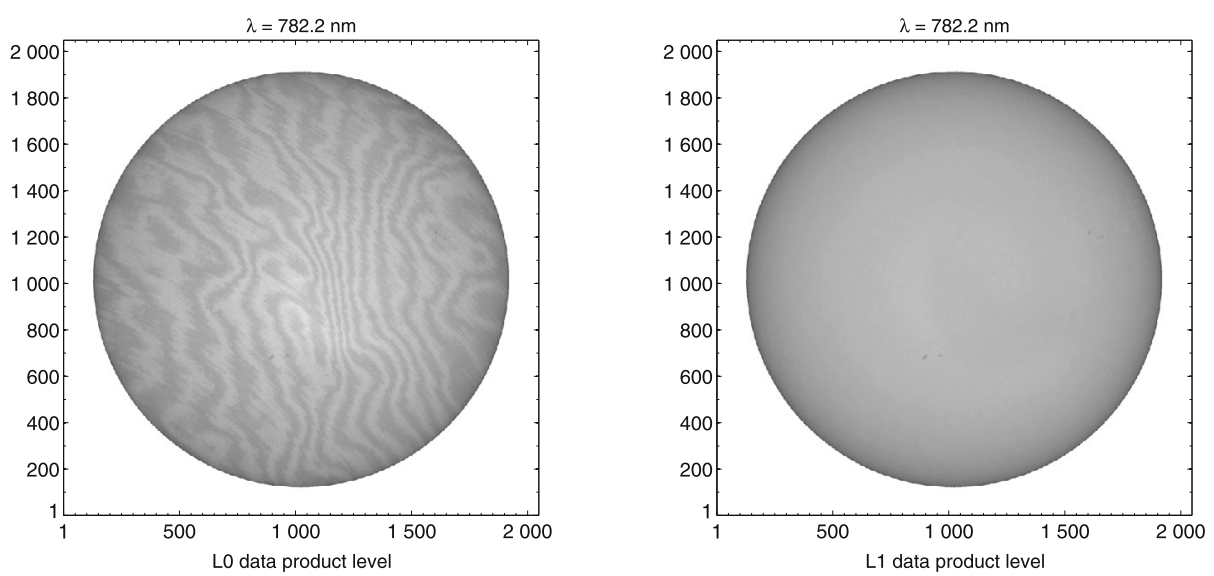

Figure 10 (Left) Raw solar image at $782.2 \mathrm{~nm}$ (Level 0 data product). (Right) Corrected solar image at $782.2 \mathrm{~nm}$ (Level 1 data product).

Table 1 Solar oblateness results $\left(\Delta_{\odot}\right)$ for two sets of measurements. $N$ represents the number of images for a series. $\Delta r_{\text {a }}$ represents the apparent equator-to-pole radius difference seen from an heliographic latitude $B_{0} . \Delta r$ is the solar equator-to-pole radius difference. $I$ represents the statistical/random uncertainty of the measurements with $95 \%$ confidence bounds $(2 \sigma) . R^{2}$ represents the goodness of fit. Rmse is the root-mean-square error of the solar oblateness fit.

\begin{tabular}{|c|c|c|c|c|c|c|c|}
\hline Date & $N$ & $\Delta r_{\mathrm{a}}$ & $\Delta r$ & $\Delta \odot$ & $I$ & $R^{2}$ & Rmse \\
\hline Sept. 2010 & 120 & 7.45 mas & $7.57 \mathrm{mas}$ & $7.89 \times 10^{-6}$ & $\pm 0.35 \mathrm{mas}$ & 0.89 & $1.5 \mathrm{mas}$ \\
\hline May 2011 & 696 & $7.82 \mathrm{mas}$ & $7.84 \mathrm{mas}$ & $8.17 \times 10^{-6}$ & $\pm 0.29 \mathrm{mas}$ & 0.92 & $1.4 \mathrm{mas}$ \\
\hline
\end{tabular}

Until now, we have focused on two sets of measurements: the first obtained during the commissioning phase in September 2010, the last obtained after a modification in the solar oblateness procedure in May 2011, when we decided to concentrate on one wavelength and increased the number of images. Between these two dates, several other intermediate measurements were made, which consist of 120 images for each position of the spacecraft, similar to the series obtained in September 2010. The results of all the sequences are shown in Figure 11. They are consistent throughout, even though their error bars are slightly greater. A comparison between the total solar irradiance (TSI) variability obtained with Picard (Meftah et al., 2014b) and the solar equator-to-pole radius difference (Figure 11) does not reveal any correlation, which is consistent with the results of Kuhn et al. (2012) and seems to respond to an issue raised in the past (Dicke, Kuhn, and Libbrecht, 1987), but when the total solar irradiance is very perturbed, the uncertainty of the measurement slightly increases. The measurement uncertainty of the solar equator-to-pole radius difference increases over time due to the aging of the instrument.

However, we consider that the best determinations of $\Delta r$ are obtained at the beginning of the mission and in May 2011, mainly because of the increased number of images and because the instrument took images for any given spacecraft roll step within the same orbit. Moreover, the stability of the instrument is preserved because there is no mechanical change during the whole sequence. The reference value of $\Delta r=7.84 \pm 0.29$ mas of May 2011 (with $2 \sigma$ errors and after correcting the heliographic latitude $B_{0}$ ) is compatible with the weighted mean value of the six analyzed sequences $(\Delta r=7.86 \pm 0.32$ mas at one $\sigma)$. 

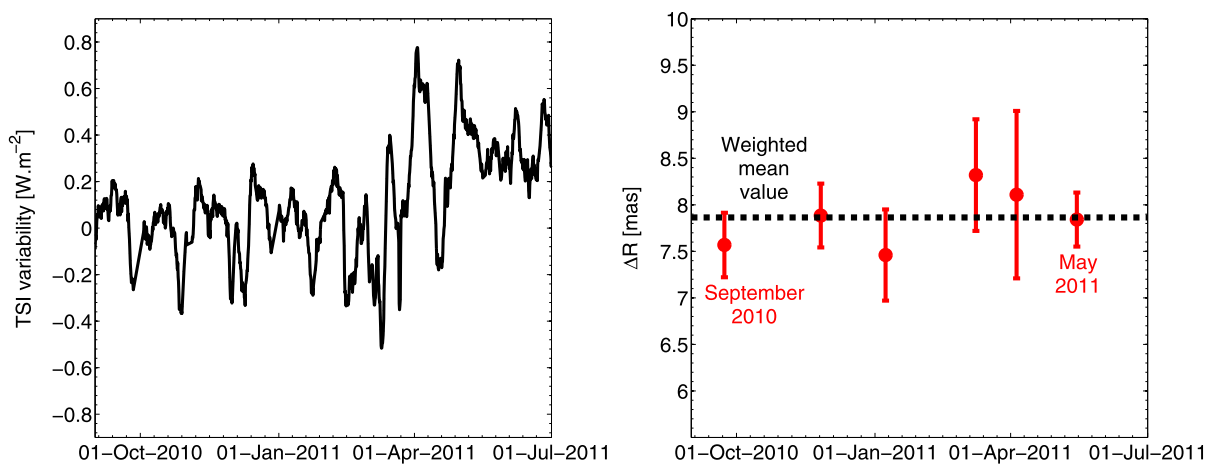

Figure 11 (Left) Evolution of the TSI, with a significant change in March-April 2011. (Right) $\Delta r$ time series at $782.2 \mathrm{~nm}(2 \sigma)$. The weighted mean value is represented by the dotted black line. The six results for solar equator-to-pole radius difference $(\Delta r)$ are represented by red circles.

Table 2 Space-borne measurements of the solar equator-to-pole radius difference $(\Delta r)$ at one $\sigma$.

\begin{tabular}{lllll}
\hline Instrument & Wavelength $[\mathrm{nm}]$ & Date & $\Delta r$ [mas] & Reference \\
\hline SoHO/MDI & 676.78 & 1997 & $8.7 \pm 2.8$ & Emilio et al. (2007) \\
RHESSI/SAS & 670.0 & 2004 & $8.01 \pm 0.14$ & Fivian et al. $(2008)$ \\
SDO/HMI & 617.3 & $2011-2012$ & $7.2 \pm 0.49$ & Kuhn et al. $(2012)$ \\
Picard/SODISM & 535.7 & 2011 & $8.4 \pm 0.3$ & Irbah et al. (2014) \\
Picard/SODISM & 782.2 & $2010-2011$ & $7.86 \pm 0.32$ & This study \\
\hline
\end{tabular}

The solar equator-to-pole radius difference we derive is too small to change the Mercury orbit outside the bounds of the general theory of relativity. Our results require a comparison with the solar equator-to-pole radius difference obtained by other space instruments. The first measurement of the solar oblateness was obtained in space with the Michelson Doppler Imager (MDI) onboard the Solar and Heliospheric Observatory (SoHO). For MDI, at a wavelength of $676.78 \mathrm{~nm}$, the solar equator-to-pole radius difference (in 1997) was $8.7 \pm 2.8$ mas (Emilio et al., 2007). Kuhn et al. (1998) reported a lower value, but we prefer to stay with the result associated with the most recent reference for the same instrument measurement. Since then, other space instruments have made this measurement: the Reuven Ramaty High Energy Solar Spectroscopic Imager (RHESSI) with its Solar Aspect Sensor (SAS), and the Solar Dynamics Observatory - Helioseismic and Magnetic Imager (SDO/HMI). As explained in the previous sections, the measurements of the solar oblateness are difficult to obtain and can reveal other phenomena, such as the additional impact of the magnetic field under the surface that affects the equatorial and polar diameters differently. Space-borne solar equator-to-pole radius difference measurements have different systematic uncertainties and have yielded different values (Table 2).

To compare the measurements, it is necessary to emphasize the wavelength of the instrument. There are probably other sources of uncertainty that are not taken into account in most of the analyses (knowledge of the systematic uncertainties, where flatfield is an example of a systematic bias). In fact, different articles obtain results that differ by more than the uncertainty error. Table 2 shows that the values obtained in space are becoming closer and are marginally consistent. The space-borne solar oblateness measurements $\left(\Delta_{\odot}\right)$ are consistent with the values proposed in theoretical models. The results obtained at $782.2 \mathrm{~nm}$ with 
our measurements are close to those found by Fivian et al. (2008). At $782.2 \mathrm{~nm}$, the more conservative $\Delta r$ value is close to $7.86 \pm 0.32$ mas at one $\sigma$ (mean of the measurements obtained by the SODISM instrument from September 2010 to May 2011). Thus, the most realistic solar oblateness value is close to $(8.19 \pm 0.33) \times 10^{-6}$, for a given wavelength. The precise measurement of the solar oblateness is still a current issue and represents to this day a scientific and technological challenge!

\section{Conclusions}

SODISM measurements of the solar shape during special roll maneuvers of the spacecraft have produced a precise determination of the solar oblateness. The raw images have revealed some optical aberrations that blur the images increasingly with time (Figure 1). In addition, the SODISM solar radius measurement evolves with time and displays a modulation in phase with the orbit, which indicates that the variations are not stochastic. Consequently, the solar oblateness procedure has been improved during the mission to achieve each roll step of the spacecraft within an orbit ( $~ 58$ images at $782.2 \mathrm{~nm}$ per step) and to take into account the thermal effects of the instrument. Thus, this procedure has allowed us to obtain good-quality data (number of images, image quality, stability, etc.).

At $782.2 \mathrm{~nm}$, the solar radius at $1 \mathrm{AU}$ is about 959.89 arcseconds $(696178 \mathrm{~km})$, which is consistent with previous results (Meftah et al., 2014c; Hauchecorne et al., 2014). This value results from the plate scale obtained during the transit of Venus (Meftah et al., 2014c). After correcting the measurements for optical distortion and for instrument temperature trend, we found a solar radius difference $(\Delta r)$ of $7.84 \pm 0.29$ mas $(2 \sigma)$. This is our reference value obtained in May 2011, with a sufficient number of images and taking orbital effects into account. Our result is close to 8 mas and agrees well with the measurements made by the RHESSI/SAS instrument. Thus, the solar oblateness value $\left(\Delta_{\odot}\right)$ is close to $(8.19 \pm 0.33) \times$ $10^{-6}$. At $782.2 \mathrm{~nm}$, the SODISM $\Delta r$ value is close to $7.86 \pm 0.32$ mas $(5.70 \pm 0.23 \mathrm{~km})$ at one $\sigma$ (mean of the measurements obtained from September 2010 to May 2011) to take into account the systematic errors with confidence. Indeed, the remaining systematic errors are a challenge for this type of measurements. Moreover, there does not appear to be any correlation with the total solar irradiance variations, considering that the solar oblateness variation, if it exists, is included within the limits of the uncertainty. This remains consistent with the results obtained by the SDO/HMI instrument. Indeed, the analysis of the HMI data indicates that the solar oblateness has remained roughly constant.

We have focused only on the solar oblateness measurements obtained in space. But a solar oblateness of $(8.63 \pm 0.88) \times 10^{-6}$, obtained from a balloon flight (Sofia, Heaps, and Twigg, 1994), is relevant and consistent with the values we have found. The measured oblateness gives an estimate of the solar gravitational moment, J2. This result is slightly lower than theoretical predictions, like the most recent space-borne results, so its accuracy is particularly interesting and challenging.

The Picard mission has reached the end of its lifetime. We hope that the SDO mission continues its measuring campaign to obtain the evolution of the solar oblateness during a full solar cycle.

Acknowledgements Picard is a mission supported by the French national center for scientific research (CNRS/INSU), by the French space agency (CNES), by the French Atomic Energy and Alternative Energies Commission (CEA), by the Belgian Space Policy (BELSPO), by the Swiss Space Office (SSO), and by the European Space Agency (ESA). The authors thank the referees for the improvements, remarks, and suggestions. 
Open Access This article is distributed under the terms of the Creative Commons Attribution License which permits any use, distribution, and reproduction in any medium, provided the original author(s) and the source are credited.

\section{References}

Brans, C., Dicke, R.H.: 1961, Mach's principle and a relativistic theory of gravitation. Phys. Rev. 124, 925. DOI.

Damiani, C., Rozelot, J.P., Lefebvre, S., Kilcik, A., Kosovichev, A.G.: 2011, A brief history of the solar oblateness. A review. J. Atmos. Solar-Terr. Phys. 73, 241. DOI.

Dicke, R.H., Goldenberg, H.M.: 1967, Solar oblateness and general relativity. Phys. Rev. Lett. 18, 313. DOI.

Dicke, R.H., Kuhn, J.R., Libbrecht, K.G.: 1987, Is the solar oblateness variable? Measurements of 1985. Astrophys. J. 318, 451. DOI.

Duez, V., Mathis, S., Turck-Chièze, S.: 2010, Effect of a fossil magnetic field on the structure of a young Sun. Mon. Not. Roy. Astron. Soc. 402, 271. DOI.

Einstein, A.: 1916, Die grundlage der allgemeinen Relativitätstheorie. Ann. Phys. 354, 769. DOI.

Emilio, M., Bush, R.I., Kuhn, J., Scherrer, P.: 2007, A changing solar shape. Astrophys. J. Lett. 660, L161. DOI.

Fazel, Z., Rozelot, J.P., Lefebvre, S., Ajabshirizadeh, A., Pireaux, S.: 2008, Solar gravitational energy and luminosity variations. New Astron. 13, 65. DOI.

Fivian, M.D., Hudson, H.S., Lin, R.P., Zahid, H.J.: 2008, A large excess in apparent solar oblateness due to surface magnetism. Science 322, 560. DOI.

Gough, D.: 2012, How oblate is the Sun? Science 337, 1611. DOI.

Hauchecorne, A., Meftah, M., Irbah, A., Couvidat, S., Bush, R., Hochedez, J.-F.: 2014, Solar radius determination from SODISM/Picard and HMI/SDO observations of the decrease of the spectral solar radiance during the 2012 June venus transit. Astrophys. J. 783(2), 127. DOI.

Hochedez, J.-F., Timmermans, C., Hauchecorne, A., Meftah, M.: 2014, Dark signal correction for a lukecold frame-transfer CCD. New method and application to the solar imager of the Picard space mission. Astron. Astrophys. 561, A17. DOI.

Irbah, A., Meftah, M., Hauchecorne, A., Cisse, E.h.M., Lin, M., Rouzé, M.: 2012, How earth atmospheric radiations may affect astronomical observations from low-orbit satellites. Proc. SPIE 8442, 84425A. DOI.

Irbah, A., Meftah, M., Hauchecorne, A., Djafer, D., Corbard, T., Bocquier, M., Momar Cisse, E.: 2014, New space value of the solar oblateness obtained with Picard. Astrophys. J. 785, 89. DOI.

Kuhn, J.R., Lin, H., Loranz, D.: 1991, Gain calibrating nonuniform image-array data using only the image data. Publ. Astron. Soc. Pac. 103, 1097. DOI.

Kuhn, J.R., Bush, R.I., Scherrer, P., Scheick, X.: 1998, The sun's shape and brightness. Nature 392, 155. DOI.

Kuhn, J.R., Bush, R., Emilio, M., Scholl, I.F.: 2012, The precise solar shape and its variability. Science 337, 1638. DOI.

Le Verrier, U.J.: 1859, Theorie DU mouvement de Mercure. In: Annales de l'Observatoire de Paris 5, 1.

Mecheri, R., Abdelatif, T., Irbah, A., Provost, J., Berthomieu, G.: 2004, New values of gravitational moments $\mathrm{J}_{2}$ and $\mathrm{J}_{4}$ deduced from helioseismology. Solar Phys. 222, 191. DOI.

Meftah, M., Irbah, A., Hauchecorne, A., Hochedez, J.-F.: 2013, Picard payload thermal control system and general impact of the space environment on astronomical observations. Proc. SPIE 8739, 87390B. DOI.

Meftah, M., Hochedez, J.-F., Irbah, A., Hauchecorne, A., Boumier, P., Corbard, T., Turck-Chièze, S., Abbaki, S., Assus, P., Bertran, E., Bourget, P., Buisson, F., Chaigneau, M., Damé, L., Djafer, D., Dufour, C., Etcheto, P., Ferrero, P., Hersé, M., Marcovici, J.-P., Meissonnier, M., Morand, F., Poiet, G., Prado, J.-Y., Renaud, C., Rouanet, N., Rouzé, M., Salabert, D., Vieau, A.-J.: 2014a, Picard SODISM, a space telescope to study the Sun from the middle ultraviolet to the near infrared. Solar Phys. 289, 1043. DOI.

Meftah, M., Dewitte, S., Irbah, A., Chevalier, A., Conscience, C., Crommelynck, D., Janssen, E., Mekaoui, S.: 2014b, SOVAP/Picard, a spaceborne radiometer to measure the total solar irradiance. Solar Phys. 289, 1885. DOI.

Meftah, M., Hauchecorne, A., Crepel, M., Irbah, A., Corbard, T., Djafer, D., Hochedez, J.-F.: 2014c, The plate scale of the SODISM instrument and the determination of the solar radius at $607.1 \mathrm{~nm}$. Solar Phys. 289, 1. DOI.

Paterno, L., Sofia, S., di Mauro, M.P.: 1996, The rotation of the Sun's core. Astron. Astrophys. 314, 940.

Poor, C.L.: 1905, The figure of the Sun. Astrophys. J. 22, 103. DOI.

Roxburgh, I.W.: 2001, Gravitational multipole moments of the Sun determined from helioseismic estimates of the internal structure and rotation. Astron. Astrophys. 377, 688. DOI. 
Rozelot, J.-P., Damiani, C.: 2011, History of solar oblateness measurements and interpretation. Eur. Phys. J. H 36, 407. DOI.

Rozelot, J.P., Fazel, Z.: 2013, Revisiting the solar oblateness: is relevant astrophysics possible? Solar Phys. 287, 161. DOI.

Rozelot, J.P., Godier, S., Lefebvre, S.: 2001, On the theory of the oblateness of the Sun. Solar Phys. 198, 223. DOI.

Sofia, S., Heaps, W., Twigg, L.W.: 1994, The solar diameter and oblateness measured by the solar disk sextant on the 1992 September 30 balloon flight. Astrophys. J. 427, 1048. DOI. 